\title{
Comparative analysis of highly defined proteases for the isolation of adipose tissuederived stem cells
}

\author{
Linda Pilgaard ${ }^{1}$, \\ Pia Lund ${ }^{1}$, \\ Jeppe G Rasmussen2, \\ Trine Fink $^{1} \&$ \\ Vladimir Z achar ${ }^{1,3 \dagger}$ \\ ${ }^{\dagger}$ Author for correspondence \\ ${ }^{1}$ Laboratory for Stem Cell \\ Research, Aalborg U niversity, \\ Fredrik Bajers Vej 3B \\ 9220 Aalborg, D enmark \\ 2D epartment of Pharmacology, \\ U niversity of Aarhus, \\ 8000 Aarhus C, D enmark \\ ${ }^{3}$ Tel.: +45 99407 556; \\ Fax: +45 9940 7816; \\ E-mail: vlaz@hst.aau.dk
}

Keywords: adipose-derived stem cells, collagenase, colony-forming unit, enzymatic dissociation, protease

future medicine $^{\text {porsg }}$

\begin{abstract}
Background: Before the potential of adipose tissue-derived stem cells can fully be exploited for a broad scope of tissue-engineering and cell-based therapeutical applications, an effective and reproducible method for isolation is needed. Aim: To comparatively analyze five highly defined protease formulations, Blendzyme 1-4, liberase $\mathrm{H} 1$ and a crude collagenase mixture in the course of digestion that consisted of three 1-h intervals. Methods: The resulting digests of human adipose tissue aspirates were evaluated for the yield of nucleated cells, viability and frequency of specific lineages, in particular CD90, CD34 and CD45, by flow cytometry. The functionality of the cells was assessed as to the colony-forming capacity in limiting dilution assays. Results: Based on all evaluation criteria, Blendzymes 1 and 2 and liberase $\mathrm{H} 1$ demonstrated a superior performance and highest consistency. Blendzyme 3 clearly underperformed compared with all other enzymes, and the performance of the rest of enzymes appeared erratic. As for the length of digestion, a 2-h interval appeared optimal when weighing both the yield and functionality of the cells in the stromal vascular fractions obtained from different adipose tissue samples. Conclusion: Our results demonstrate that the highly purified proteases provide a valuable alternative to crude collagenase preparations, especially in scenarios where a high definition and reproducibility of the digestion process is of importance.
\end{abstract}

Custom-designed regenerative medicine based on autologous stem cells is a rapidly growing field of research within stem cell biology. Bone marrow stromal cells (BM SCs) that comprise mesenchymal stem cells have previously proven their potential in a number of therapeutical applications [1-4]. The harvest of bone marrow demands an invasive procedure causing a significant amount of discomfort for the patient, and the number of isolated cells is restricted owing to limitations in dispensable bone marrow volume. Adipose tissue-derived stem cells (ASCs) have been characterized and found to have a differentiation potential and phenotype similar to that of BMSCS [5]. The aspiration of adipose tissue inflicts a minimum of discomfort on the patient and the volume of tissue that can be aspirated is less restricted. These benefits make ASC s highly suitable for tissue-engineering applications.

In order to implement new therapies, effective and reproducible methods for isolation and expansion of ASC s are needed. It has been shown that parameters, such as digest time and enzyme activity, applied in the isolation procedure of primary cells have a significant effect on cell yield, viability and phenotype [6-9]. In addition, in vitro culture time and conditions affect stem cell viability and differentiation potential. In vitro culture introduces increased risk of contamination and cell senescence with culture time [10-12], thus it is crucial that in order to prevent variations in protocols that may produce suboptimal cell populations, the enzymatic release of ASC s are optimized. The adipose tissue aspirate is most often digested by collagenase. Typically, either a purified type of collagenase or a crude collagenase mix (CCM) is applied. The collagenases most often originate from Clostridium histolyticum. In the case of $\mathrm{CCM}$ s, the collagenase mixture contains a variation of proteases in addition to the collagenase, and hence varies in collagenase activity and enzyme composition between the lots $[7,8,13]$. The use of purified collagenase is insufficient, as additional protease activity has proven to be essential for optimal tissue digest efficacy and cell yield [8]. The challenge is to find the best combination of enzymes and digest time, and balance these against the desired cell yield and cell function for the application. Additionally, the necessity and risk of in vitro expansion has to be considered.

In the current investigation, we set out to identify the enzyme and digestion interval to yield the highest number of cells with colonyforming capacity when isolating the ASC $\mathrm{s}$. Six different enzyme blends of equal collagenase activity but variable protease composition and activity were tested on human adipose tissue 
aspirate during the course of 1-, 2- or 3-h digestion. Although relatively long compared with the standard of 30-60 min $[10,14,15]$, these digestion intervals were chosen to exhaustively isolate the ASC s. Highly defined Blendzyme 1-4 (B1-4), as well as human liberase $\mathrm{H} 1$ (all from Roche Applied Sciences, $H$ vidovre, D enmark) that have been approved for clinical use were selected. As a reference, a CCM from $C$. histolyticum that was optimized and regularly used for ASC isolation in our laboratory was included. The resulting stromal vascular fraction (SVF) of the cells was assayed for viability and function with regard to the colony-forming capacity along with the phenotypic characteristics.

\section{Materials \& methods}

Donors \& adipose tissue sampling

Samples of subcutaneous fat were obtained after informed consent from six female patients (age: 26-45; mean: 35 years) undergoing elective surgery at the Grymer Private H ospital, Skejby, D enmark (Table 1). The liposuctions were all performed identically using the same tumescent technique [16] and pump-assisted aspiration was carried out by one surgeon. W ithin $12 \mathrm{~h}$ of collection, the adipose tissue was transported at room temperature to the laboratory and processed. All protocols have been reviewed and approved by the regional Committee on Biomedical Research Ethics in N orthern Jutland, D enmark.

Isolation of stromal vascular fraction from adipose tissue

The cells making up the adipose stroma were isolated according to a previously described protocol [14] with slight modifications. Six different protease mixtures, including B1-4, liberase $\mathrm{H} 1$ (Roche Applied Sciences) and CCM (lot no. LTQ 5230; Wako, Neuss, Germany), were employed (Table 2). The working solution for each enzyme was freshly prepared prior to use by dissolving to a final collagenase activity of

\begin{tabular}{|lcll}
\hline \multicolumn{4}{l}{ Table 1. Summary of donor data. } \\
\hline Donor ID & Age & Harvest site & BMI \\
\hline \#22 & 45 & Buttocks, inner thigh & 24.3 \\
$\# 23$ & 42 & Inner thigh & 21.0 \\
$\# 25$ & 33 & Abdomen, thigh & 25.8 \\
$\# 26$ & 26 & Abdomen, hip & 21.5 \\
$\# 27$ & 35 & Abdomen, thigh & 21.2 \\
\hline 28 & 30 & Abdomen, thigh & 21.5 \\
\hline BMI: Body mass index.
\end{tabular}

$0.28 \mathrm{~W}$ ünsch $\mathrm{U} / \mathrm{ml}$. This concentration was selected based on the activity of our collagenase reference, which also fits well within the commonly used $0.075-0.1 \%$ concentration range [17-19]. For enzymes, the concentration of which was expressed in $M$ andl units, a conversion factor $1000 \mathrm{M}$ andl units = $1 \mathrm{~W}$ ünsch was used. In the case of CCM , the enzyme solution was dissolved in Dulbecco's phosphate-buffered saline (D -PBS) containing $\mathrm{Ca}^{2+}$ and $\mathrm{M} \mathrm{g}{ }^{2+}$ (Invitrogen, Taastrup, D enmark) and supplemented with $2 \%$ bovine serum albumin (BSA) according to manufacturer instructions for optimal enzyme availability and cell stabilization. The B1-4 and liberase $\mathrm{HI}$ with included buffer salts were reconstituted in the delivery vial as recommended by the producer. $\mathrm{N} o$ additional BSA was supplemented with the latter enzymes.

The fat tissue was, prior to digestion, washed three times with equal volumes of prewarmed D-PBS, and for digestion, $10 \mathrm{ml}$ of the adipose tissue was mixed with an equal volume of enzyme buffer. The incubation proceeded at $37^{\circ} \mathrm{C}$ under gentle agitation for 1,2 or $3 \mathrm{~h}$. The dissociated tissue was fractionated by sedimentation centrifugation at $400 \mathrm{~g}$ for $10 \mathrm{~min}$ and the pelleted cells were filtered through a 70- $\mathrm{mm}$ mesh cell strainer (BD Bioscience, Broendby, Denmark) to remove debris. Contaminating erythrocytes were lyzed using sterile water and the remaining nucleated cells were further purified through a second round of centrifugation and filtration. The total yield was determined in a hemocytometer after the cells had been stained with acetic methylene violet.

Immunophenotyping \& flow cytometry The flow cytometric analysis for a mesenchymal stem cell marker, CD 90, hematopoetic progenitor cell marker, CD 34, and a leukocyte lineage marker, CD 45, was performed with freshly isolated cells in a triple immunostaining procedure to assess coexpression of the assayed epitopes. The primary antibodies were mouse monoclonals from $D$ ako (G lostrup, D enmark), CD 34 (\#M 7080), C D 45 (\#M B0742), IgG 1 (\#X 0931) and IgG2a (\#X0943) or Abcam (C ambridge, UK) and CD 90 (\#ab11153). The Zenon labeling system (Invitrogen), including Alexa Fluor 488 (\#Z25002), Alexa Fluor 647 (\#Z25008) and R-Phycoerythrin (\# Z25055), was used to discriminate the primary antibodies. The antibodies and fluorophores were combined to provide conjugates CD 34-Phycoerythrin, CD 45-Alexa Fluor 488 and CD 90-Alexa 
Table 2. Enzyme specifications.

\begin{tabular}{lll} 
Enzyme & $\begin{array}{l}\text { Collagenase activity } \\
\text { (Wünsch } \mathbf{~} / \mathbf{m l} \text { ) }\end{array}$ & $\begin{array}{l}\text { Target neutral protease } \\
\text { (caseinase } \mathbf{U} / \mathbf{m l} \text { ) }\end{array}$ \\
\hline Blendzyme 1 & 0.28 & Dispase (30) \\
\hline Blendzyme 2 & 0.28 & Thermolysin (60) \\
Blendzyme 3 & 0.28 & Thermolysin (120) \\
Blendzyme 4 & 0.28 & Thermolysin (240) \\
\hline Liberase H1 & 0.28 & NA \\
\hline Collagenase & 0.26 & $*$ \\
\hline
\end{tabular}

*Lot no. LTQ5230.

NA: Not applicable.
Fluor 647. For staining, approximately $5 \times 10^{5}$ cells were reacted with antibodies appropriately diluted in stain buffer (BD Bioscience) at room temperature for $1 \mathrm{~h}$. After incubation, the cells were washed and resuspended in stain buffer and kept on ice until analysis. The isotype controls were applied to correctly control for corresponding antibodies.

Before flow cytometric analysis, the instrument acquisition parameters were calibrated using FAC S 7 Color Beads (BD Biosciences) and compensation settings were adjusted using stained and unstained cultured ASCs. For each sample, $3 \times 10^{4}$ events were acquired with a FACSC anto ${ }^{\text {tm }}$ flow cytometer (BD Bioscience), and analyzed using BD FACSDiVa ${ }^{\text {Tm }}$ (BD Bioscience) and Flowjo (TreeStar, Ashland, OR, USA) software packages. Cells were gated using forward and side scatters to eliminate cell debris and to select the P1 population for analysis. Within the $\mathrm{P} 1$ population, a population $\mathrm{P} 2$ positive for a given epitope was defined as having less than $1 \%$ false-positive events from autofluorescence seen in unstained cells or nonspecific binding in isotype control. For each staining, the percentage of cells in the $\mathrm{P} 2$ population was determined, and using the total yield of nucleated cells subsequently translated into the yield of cells from $\mathrm{ml}$ of fat tissue.

\section{Viability assay}

To assess the viability of freshly isolated cells, the LIVE/DEAD ${ }^{\circledR}$ Reduced Biohazard Viability/Cytotoxicity Kit (Invitrogen) was employed. The assay was based on the nucleic acid stains Syto 10 green, which labels all cells, and the DEAD Red, which stains only cells with compromised cytoplasmic membrane. Approximately $5 \times 10^{5}$ cells were treated according to manufacturer's instructions, after which the analysis was carried out with FACSCanto flow cytometer using 515-545- and 564-606-nm emission channels. The calibration, compensation and gating parameters were used as described previously.

\section{Cell cultures}

Cells were cultured in growth medium, Dulbecco's M odified Eagle M edium and nutrient mixture F-12 (Invitrogen) supplemented with $10 \%$ fetal calf serum and antibiotics, including penicillin, streptomycin and gentamicin. All cultures were kept in a humidified atmosphere containing $5 \% \mathrm{CO}_{2}$ buffered with ambient air at $37^{\circ} \mathrm{C}$. The medium was changed twice a week.

\section{Colony-forming unit assays}

To assess the frequency of colony-forming units (CFUs) among the isolated nucleated cells, a limiting dilution assay was performed. The freshly isolated cells were suspended in growth medium at a concentration of 50,000 cells $/ \mathrm{ml}$. A serial twofold dilution across the 12 columns of a 96-well cell culture plate was performed, thus resulting in rows containing from $4-10^{4}$ cells per well. For each enzyme and digestion interval combination, one 96-well plate was prepared to assay for colony-forming unit fibroblasts (CFU-Fs). The cells were incubated for 11 days in the growth medium, at which point the plates were fixed with $4 \%$ formaldehyde and stained with methylene blue. The number of empty wells, which were the wells found to contain colonies of less than 20 methylene blue positive cells, was determined for each dilution series. The scoring was carried out independently by two observers.

The data from the limiting dilution assay was used to calculate the number of CFU -Fs based on the Poisson distribution according to the equations $F_{0}=e^{-u}$ and $u=-\operatorname{lnF}_{0}$, where $F_{0}$ is the fraction of empty wells within the dilution series and $u$ is the average number of CFU s per well [20-22]. The u value was calculated for each dilution series and the average number of CFU-Fs was determined. Based on the total yield of nucleated cells per $\mathrm{ml}$ of adipose tissue, the absolute yield of CFU-Fs was calculated for individual enzymes and digestion times.

\section{Statistics}

The data are presented as arithmetic means \pm standard error of mean. N onparametric statistics were used to test for differences between populations. M ultiple related samples were compared using the Friedman test, the results of which were complemented with post-hoc pairwise 
analysis by Wilcoxon signed-rank test. The routines were carried out with the aid of the SPSS v.14 software package (SPSS, C hicago, IL, USA) and the statistical significance was assigned to the differences at $p<0.05$.

\section{Results}

Viability \& phenotypic analysis of crude adipose tissue digests

A representative distribution of analyzed subpopulations within crude SVFs is presented in

Figure 1A. The forward versus side scatter was used to discriminate from cell debris. The gated population (P1) comprised approximately $80 \%$ of all events, approximately $10 \%$ of which constituted dead cells. In order to correctly determine the proportion of $\mathrm{CD} 34^{+}, \mathrm{CD} 90^{+}$and $\mathrm{CD} 45^{+}$cells, the range of specific signal intensities was determined from the background staining by isotype control relevant for each of the specific antibodies. Since the experimental procedure allowed for a simultaneous identification of all three epitopes, bivariate plots representing a measure of CD 90 and CD 34, and CD 90 and CD 45 coexpression were possible.

As to the viability of isolated cells, approximately $90 \%$ survived the procedure, based on an average across all enzymes and digestion times (Figure 1B). There was only one protease, B1, which resulted in a significantly lower proportion of live cells, irrespective of the length of digestion period. The same enzyme, however, yielded a significantly higher proportion of specific precursors, CD $90^{+}$and $C D 34^{+}$. In general, the percentage of live cells in general did not change significantly with longer incubation time. By contrast, the relative yields of CD 90and $C D 34^{+}$cells increased significantly with time. The percentage of leukocyte lineage cells did not change with prolonged digestion. This fraction represented on average $3.4 \%$ of the total yield of nucleated cells in the SVF. O verall, it is striking to notice the broad range of the obtained relative yields that underscores the existence of remarkably high interdonor variability.

Total \& phenotype-specific cell yields Q uantitative data pertaining to the total and phenotype-specific cell yield are displayed in Figure 2. The total yield of freshly isolated nucleated cells was determined in six donor cases for each combination of enzyme and digestion time (Figure 2A). Looking at the individual enzymes, B3 consistently yielded lower numbers at all three digestion times. $\mathrm{N}$ onetheless, the difference in total cell yield was not significant between enzymes within each of the three evaluated digestion periods. Interestingly, the total cell yield increased significantly with the digestion time. Disregarding the applied enzyme, the overall average yield of nucleated cells increased from $1.7 \times 10^{5}$ at the first hour to $4.0 \times 10^{5}$ cells per $\mathrm{ml}$ adipose tissue after $3 \mathrm{~h}$ of digestion. As to the potential to recover viable cells, a significant difference was observed between the enzymes. B2 and the CCM yielded significantly higher numbers of viable cells at all time points as compared with B3 and 4.

The duration of digestion also had a significant impact, as evident from differences between cell yields at the first and last hour of incubation.

With regard to the yield of CD $90^{+}$cells, no single enzyme could be indentified as significantly outperforming or underperforming, although B3 was clearly found trailing performances of competitor proteases (Figure 2B). When taking into account the duration of digestion, the yield had a tendency to increase with time. Specifically, this increase was significant for B2 and collagenase, and it corresponded to more than a threefold rise during an interval from the first to the third hour. Regarding the yield of CD $34^{+}$cells, there was no significant difference when comparing the performance of individual enzymes at different digestion times (Figure 2B ). It is noteworthy that for B1-3 and collagenase, a significant increase in the yield of CD $34^{+}$cells was obtained by prolonging the digestion from 1-3 h. W hen taking into account all enzymes, the yield increase was more than threefold and corresponded to a rise from $5300 \mathrm{cell} / \mathrm{s} / \mathrm{ml}$ of adipose tissue in the first hour to approximately 18,000 cells $/ \mathrm{ml}$ of fat sample at the completion of digestion. The yield of CD $45^{+}$cells represented only a nonsignificant contamination during the digestion procedure (Figure 2B), which is consistent with only a very low proportion of these cell in the SVF (Figure 1A \& B ).

Yield of nonspecific colony-forming precursors

The functionality of the isolated nucleated cells was tested by their capacity to support outgrowth of colonies in a limited dilution assay. Among the enzymes, there was a significant difference in CFU-F yield, both when comparing within the individual digestion times and irrespective of digestion periods (Figure 2C). B1 and collagenase yielded the highest numbers of $\mathrm{CFU}-\mathrm{Fs}$, all significantly higher than the worst-performing 


\section{Figure 1. Flow cytometric analysis of stromal vascular fractions.}

A

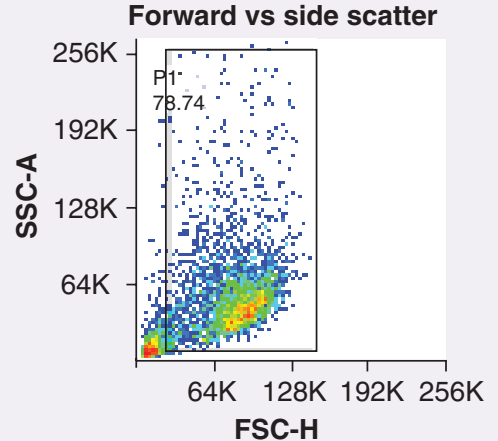

CD90

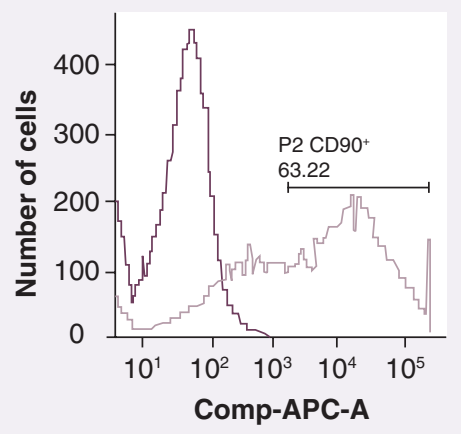

CD90 vs CD34

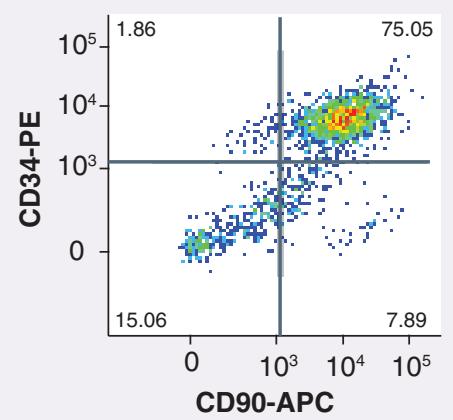

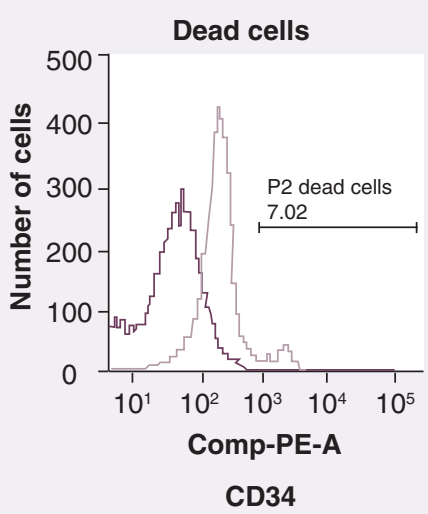

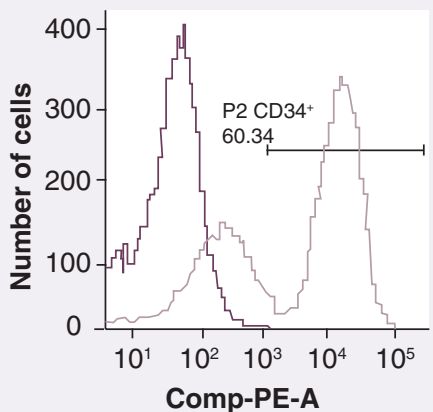

CD90 vs CD45

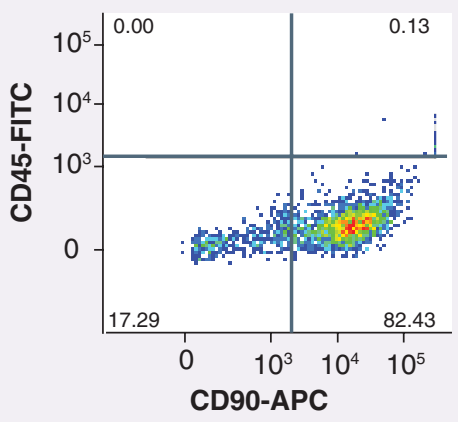

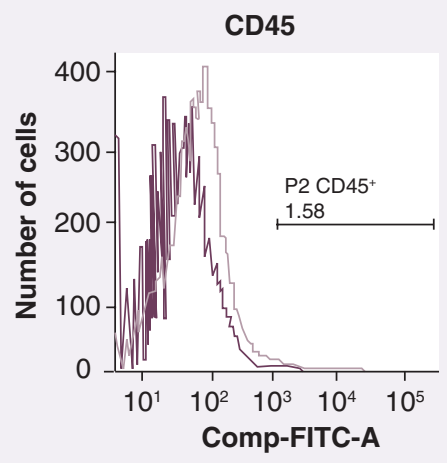

\begin{tabular}{|lllll|}
\multicolumn{5}{c}{ Cell yield by time (\%) } \\
\hline Time & Live & CD90+ & CD34+ & CD45+ \\
\hline \multirow{2}{*}{$1 \mathrm{~h}$} & 88.0 & 42.4 & 33.5 & 3.1 \\
& $(42.1-99.6)$ & $(6.5-93.2)$ & $(1.8-89.6)$ & $(0.1-15.5)$ \\
& & &
\end{tabular}

\begin{tabular}{|c|c|c|c|c|}
\hline $2 \mathrm{~h}$ & $\begin{array}{l}89.9 \\
(41.9-99.8)\end{array}$ & $\begin{array}{l}42.4 \\
(11.2-70.2\end{array}$ & $\begin{array}{l}35.3 \\
(1.4-71.9)\end{array}$ & $\begin{array}{l}4.7 \\
(0.1-28.6)\end{array}$ \\
\hline & $\begin{array}{l}92.0 \\
(42.1-99.4)\end{array}$ & $\begin{array}{l}47.9^{*} \\
(8.1-75.7)\end{array}$ & $\begin{array}{l}43.6^{*} \\
(2.1-70.8)\end{array}$ & $\begin{array}{l}2.3 \\
(0.1-13.2)\end{array}$ \\
\hline
\end{tabular}

(A) Forward and side-scatter characteristics and frequency distributions together with bivariate plots for selected markers from a representative donor. Light purple lines indicate a specific signal, whereas dark purple lines refer to isotype controls. (B) Relative cell yields and ranges (maximum and minimum) are categorized according to enzyme type (left) or digestion time (right). * Denotes a significant difference between enzyme types or digestion times that provided a highest and lowest yield in a given category $(p<0.05)$. 


\section{Figure 2. Absolute yield of cells and colony-forming units fibroblasts.}

A

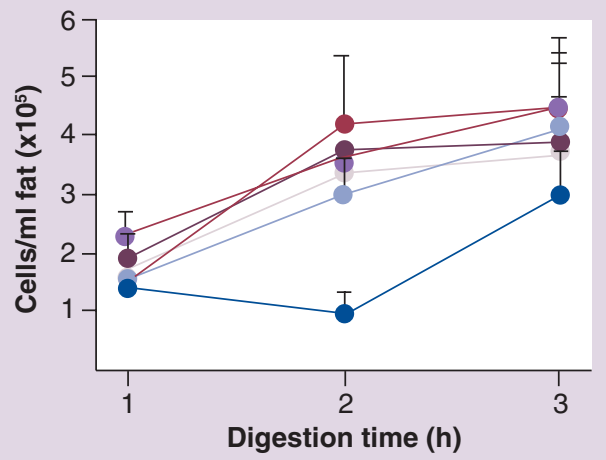

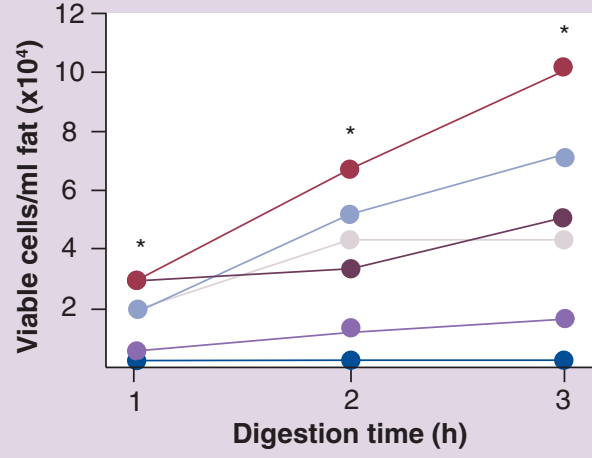

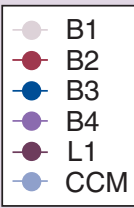

B

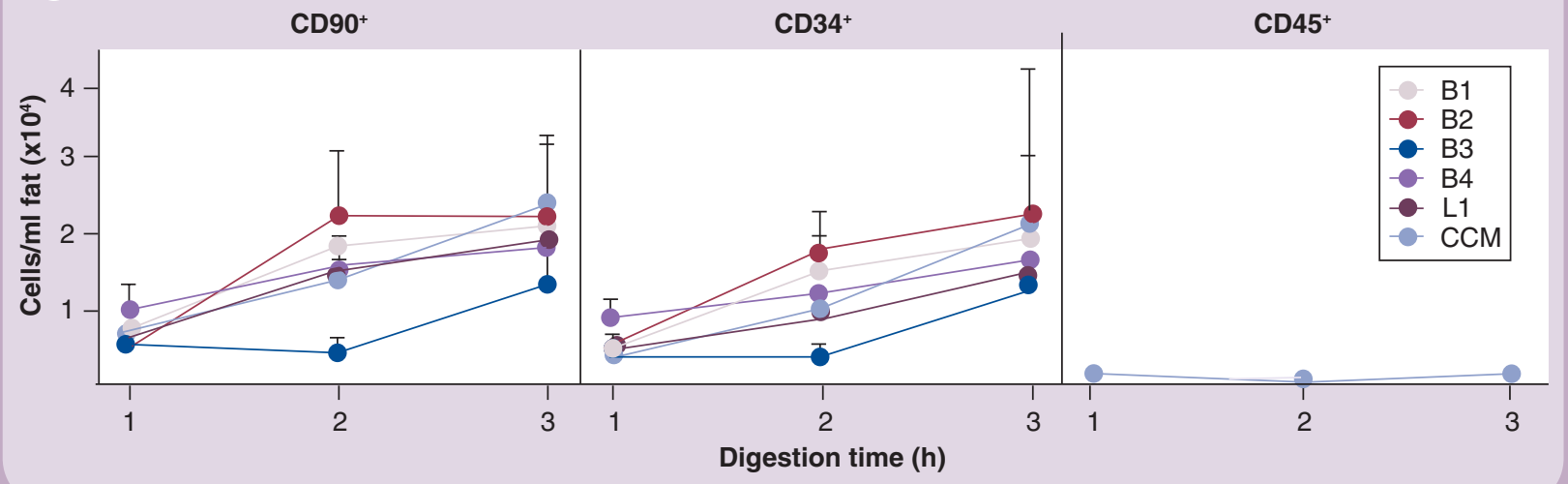

C

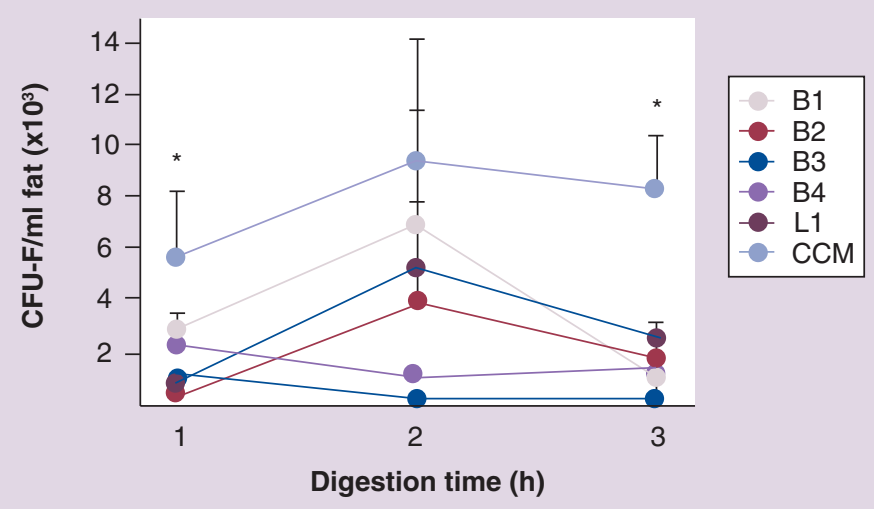

(A) Total yield of nucleated and viable cells per $\mathrm{ml}$ of lipoaspirate. (B) Total yield of specific cell lineages per $\mathrm{ml}$ of lipoaspirate.

(C) CFU-F per $\mathrm{ml}$ of lipoaspirate. Error bars denote standard error of mean for the number of donors included ( $\mathrm{n}=6$, owing to reduced tissue volume; $\mathrm{n}=5$ for CFU-F).

* Denotes a significant difference between the best and worst performing enzymes at a given digestion point $(p<0.05)$.

B: Blendzyme; CCM : Crude collagenase mix; CFU-F: Colony-forming units fibroblast; L1: Liberase H1.

enzyme, B3. These two best-performing enzymes, when taking into account all digestion intervals, yielded averages of 3500 and $7800 \mathrm{CFU}-\mathrm{Fs} / \mathrm{ml}$ adipose tissue corresponding to 2 and $1.5 \%$ of the total yield of nucleated cells, respectively. As for the length of digestion, time did not appear to have a significant effect on the yield of CFU-F/ml adipose tissue. 
Yield ranks by enzyme type

$\&$ digestion time

When comparing the enzymes efficacy at dissociating the fat tissue, it is striking that for each individual donor a highly distinct performance ranking was determined. The performance of enzyme preparations was evaluated by ranking their respective cell yields from six independent tissue samples/donors (for the CFU - F assay five donors were available) and during three digestion intervals. The statistical significance regarding the enzyme performance or incubation time was calculated using appropriate tests. Figure $3 \mathbf{A}$ represents the enzyme rankings within three categories that are most pertinent to the isolation of potentially multipotent ASCS. The rankings take into account average values from all digestion intervals. B3 clearly underperformed all other enzymes. $\mathrm{B} 1$ and 2 , and liberase $\mathrm{H} 1$, on the other hand, appeared to outperform the other enzymes in overall score but also seemed to maintain a balanced performance across all categories. The performance of the rest of

\section{Figure 3. Enzyme type- and digestion time-specific ranks.}

A

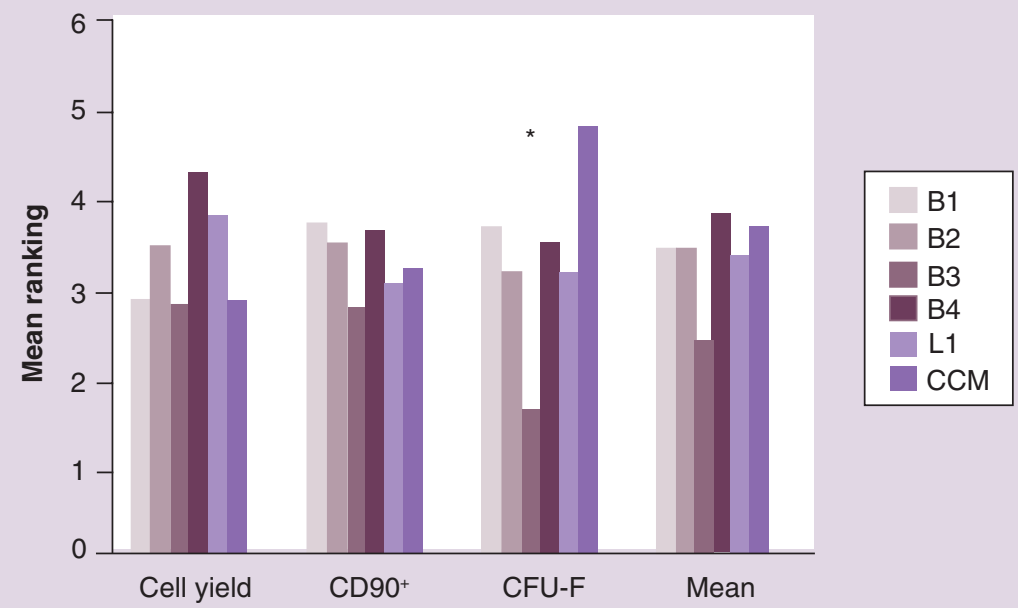

B

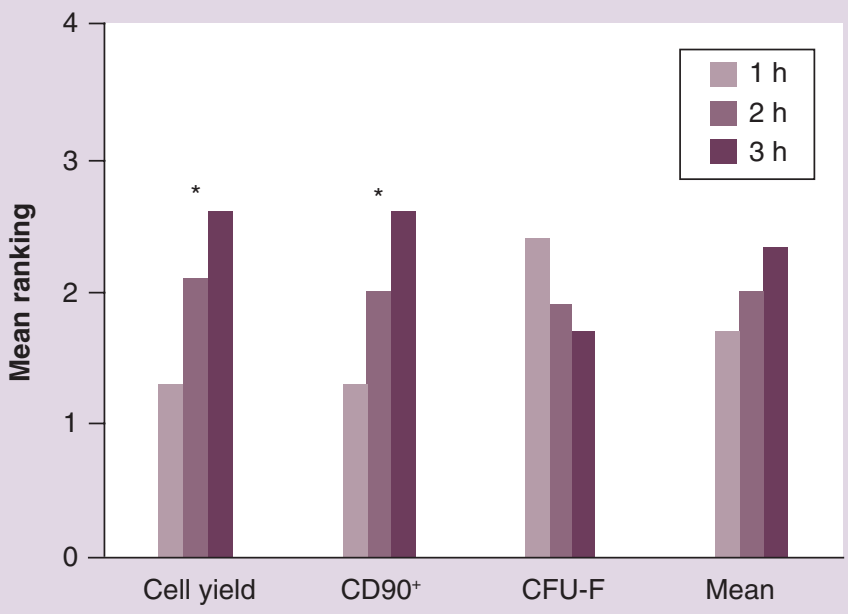

(A) M ean ranks of all enzyme types regardless of digestion time for selected categories. (B) M ean ranks of all digestion intervals regardless of enzyme type for selected categories. * Denotes a significant difference between the top and bottom ranking digestion interval at a given category $(p<0.05)$.

B: Blendzyme; CCM : Crude collagenase mix; CFU-F: Colony-forming units fibroblast; L1: Liberase H1. 
enzymes appeared more erratic. It is important to note, however, that the ranking order could not be supported at a statistically significant level. In Figure 3B, the ranking of digestion intervals, which take into account average values from all enzymes, is listed. Although it is apparent that extended digestion yields significantly more nucleated as well as CD $90^{+}$cells, there is no evidence that such correlation also applies to the yield of CFU-F. Considering the average score, it seems that 2-h incubation would be the most suitable interval to provide for optimal yields and CFU - F numbers.

\section{Discussion}

In the current investigation, we set out to standardize the enzymatic dissociation step of the isolation procedure for adipose-derived mesenchymal stem cells. Previous investigations have accumulated evidence with regard to a substantial interindividual variability of SVF parameters, such as total cell yield or phenotypical and functional characteristics. The great variability is assumed to arise from specifics in donor demographics, such as body mass index and age $[11,16,17,23]$. 0 ur own data confirm great discrepancy in enzyme performance between different individuals. In order to minimize the individual specific factors, we ranked the data for donors separately and subsequently used the average ranking across donors to test for the significance of the relevant analysis result. Friedman's statistical routine was conveniently used for this purpose. Since the approach also accommodates for repeated measurements, it was possible to simultaneously take into account different digestion intervals and thus increase the power of analysis. O ur comprehensive comparison of six different enzyme preparations indicates that B1 and 2 , and liberase $\mathrm{H} 1$ are most universal when a broad spectrum of goals, ranging from a quantitative yield of cells to the yield of biologically active progenitors, is to be met. The analysis of digestion times revealed that although the yield of specific cell types increases with time, this is not true for biologically active precursors. Thus, it appears that for this set-up a digestion period of $2 \mathrm{~h}$ would satisfy most of the experimental demands.

In the present study, we implemented longer digestion intervals than traditionally used and additionally, the enzyme activity was lower than that commonly used in protocols based on shorter intervals and higher enzyme concentrations. The set-up was inspired by the work of
Wang et al. who found that although the fre quency of cultivable dermal fibroblast and their proliferation rate decreased with time, the total cell outcome was elevated owing to the increase of the cell yield [7]. In order to exhaustively isolate the ASC population, we opted to prolong the digestion period threefold. The more effective tissue dissociation was apparent from the increase in the relative as well as absolute cell yields. The higher yield of both CD $90^{+}$and CD $34^{+}$cells indicates a more efficient cell release in general, and in the case of the hematopoietic progenitors, a progressive disintegration of tissue microvasculature.

Conversely, the low yield of CD $45^{+}$leukocyte lineage, reaching consistently around $3.4 \%$ of the total yield of nucleated cells in the SVF, confirms that these cells are not associated with the extracellular matrix of the adipose tissue. O ur results are in agreement with some $[17,24,25]$ but not all [18] of the previous studies. The discrepancy is likely to reflect differences between the tissue samples themselves, since the anatomical sites differ with respect to vascularization, tissue harvest methods and isolation procedures.

Regarding the biological activity of isolated cells, the capability of supporting the outgrowth of colonies (CFU-F) averaged $1 \%$ of the SVF pool in our study. This number was very similar for all enzymes as well as digestion periods, and was also in agreement with previously published figures [11]. W ith the stable fraction of approximately $90 \%$ viable cells, this result was expected. It is interesting that the low yield of CFU-Fs is in contrast with the high frequency of progenitor $\mathrm{CD} 90^{+}$cells, which in addition appear to assume an increasing fraction during the course of digestion.

Similar results were previously demonstrated by 0 edayrajsingh-Varma et al., where three tissue harvest methods yielded similar numbers of viable cells but differed in the frequency of ASC s [19]. An explanation could be the late onset of apoptosis caused by the extended exposure to proteolytic enzymes. The LIVE/DEAD assay employed in current investigation is only able to distinguish between intact cells and cells with compromised membrane integrity. In addition, the SVF cells were plated for the CFU -F assay according to the number of live cells based on trypan blue staining, which identifies dead cells with compromised membranes. Sufficient amounts of stress and/or DNA damage as a result of the enzyme treatment combined with removal from the natural 
microenvironment could initiate an apoptotic reaction in the SVF cells. Provided the presence of a very initial phase of apoptosis, the results in the CFU $-F$ assay may have been affected, since the used methods are not effective in such a scenario. Further analysis would be necessary to determine a relationship between enzymatic treatment, culture conditions and the occurrence of apoptosis.

Despite the increased cell yield with prolonged digestion, the results indicate that the cell source had not been fully exhausted from cells, as no plateau in cell yields was reached. Hence, more progenitor cells could potentially be isolated. The challenge is to isolate the maximum number of functional progenitors without damaging the cell population in the process. To achieve this, one could vary the combination of enzyme activity with different digestion periods. Inferring from the interindividual variability, the ideal set-up would include a test to determine the most optimal enzyme formulation in each case. Subsequently, a long-term digest would be performed in a sequential manner, where released cells are retrieved continuously, thus avoiding proteolytic overexposure.

The main goal of the current study was to comparatively analyze a complement of highly defined proteases with the crude collagenase type I enzyme, which hitherto was a mainstay tool to obtain SVFs. Surprisingly, such an investigation has not been carried out before, but with the prospect of exploiting of ASC s in clinical applications [26], it appears critical that highly reproducible protocols are developed. Crude collagenase, unfortunately, is subject to a substantial lot-to-lot variation and standardization is hardly achievable, in addition to the fact that its composition is too poorly defined to conform with good manufacturing practice standards. N evertheless, it is interesting that the highly defined enzymatic formulations did not significantly outperform crude collagenase from our study, or for that matter in the previous investigations, as far as the yield of precursors is concerned $[10,11,14-17,22,23]$. M oreover, the interindividual variability appeared to be of such a magnitude that the data to identify the best-performing enzyme with a statistical significance could not be obtained. Thus, despite the fact that the new generation of enzyme mixes does not offer substantial benefit over the commonly used collagenase with regard to the quantitative yields, the purified proteases still appear to be an appealing alternative maybe in a new sequential approach and especially in cases where the digestion process needs to be highly defined and reproducible.

\section{Conclusion}

In the current investigation, five highly defined protease formulations and a CCM were comparatively evaluated at 1, 2 and $3 \mathrm{~h}$ of digestion. The resulting SVFs of human adipose tissue aspirates were analyzed for the yield of nucleated cells, viability and frequency of specific lineages by flow cytometry. The functionality of the cells was assessed as to the colony forming capacity in

Executive summary

\section{Key findings}

- The forward versus side-gated population within crude stromal vascular fraction cells (P1) comprised approximately $80 \%$ of all events and $90 \%$ of the cells were viable. The total cell yield increased significantly with the digestion time.

- The relative yields of $\mathrm{CD} 90^{+}$and $\mathrm{CD} 34^{+}$cells increased significantly with time, whereas the percentage of leukocyte lineage cells represented only a nonsignificant contamination during the digestion procedure and did not change with prolonged digestion.

- Blendzyme 2 and the crude collagenase mix yielded significantly higher numbers of viable cells at all time points as compared with Blendzymes 3 and 4.

- Blendzyme 1 and crude collagenase yielded the highest numbers of colony-forming unit fibroblats, all significantly higher than the worst performing enzyme, the Blendzyme 3.

\section{Condusions}

- Blendzymes 1 and 2, and liberase H1, are most universal when a broad spectrum of goals, ranging from a quantitative yield of cells and potential precursor to the yield of biologically active progenitors, is to be met.

- The analysis of digestion times revealed that a period of $2 \mathrm{~h}$ would satisfy most of the experimental demands.

- The highly purified proteases provide a valuable alternative to crude collagenase preparations, especially in scenarios where high definition and reproducibility of the digestion process is of high priority.

- By using selected formulations of highly defined protease mixtures, along with well-tuned growth conditions, an adipose tissue-derived stem cell population highly suitable for regenerative clinical applications may readily become available. 
a limiting dilution assay. $\mathrm{O}$ ur data provide evidence that $\mathrm{B} 1$ and 2 , and liberase $\mathrm{H} 1$ outperform other enzyme preparations, whereas B3 clearly trails in performance. As for the length of digestion, a 2-h interval appeared optimal when considering both the yield and functionality of the cells in the SVF. In conclusion, our results demonstrate that the highly purified proteases provide a valuable alternative to crude collagenase preparations, especially in scenarios where high definition and reproducibility of the digestion process is of high priority.

\begin{abstract}
Acknowledgements
The authors wish to thank plastic surgeons Frants Grymer and Christian Bang, their office and nursing staff, and their patients at Grymer Private H ospital, Aarhus, Denmark, for donation of the liposuction material. The expert technical assistance of $\mathrm{H}$ elleSkjødt M øller is highly appreciated.
\end{abstract}

Financial \& competing interests disclosure

The authors greatly appreciate support from the $C$ arlsberg, John and Birthe M eyer's, Toyota Foundations and the $D$ anish M edical Research Council grants no. 271-06-0283 and 2052-01-0045. The authors have no other relevant affiliations or financial involvement with any organization or entity with a financial interest in or financial conflict with the subject matter or materials discussed in the manuscript apart from those disclosed.

No writing assistance was utilized in the production of this manuscript.

Ethical conduct of research

The authors state that they have obtained appropriate institutional review board approval or have followed the principles outlined in the Declaration of $\mathrm{H}$ elsinki for all human or animal experimental investigations In addition, for investigati ons involving human subjects, informed consent has been obtained from the participants involved.

\section{Bibliography}

Papers of special note have been highlighted as either of interest $(\bullet)$ or of considerableinterest $(\bullet)$ to readers.

1. Wollert KC, M eyer GP, Lotz J et al.: Intracoronary autologous bone-marrow cell transfer after myocardial infarction: the BO OST randomised controlled clinical trial. Lancet 364, 141-148 (2004).

2. H orwitz EM, Prockop DJ, Fitzpatrick LA et al.: Transplantability and therapeutic effects of bone marrow-derived mesenchymal cells in children with osteogenesis imperfecta. $\mathrm{N}$ at. M ed. 5 , 309-313 (1999).

3. Aviles FF, San Roman JA, Garcia Frade J et al.: Intracoronary stem cell transplantation in acute myocardial infarction. Rev. Esp. Cardiol. 57, 201-208 (2004).

4. Kuroda $\mathrm{R}$, Ishida $\mathrm{K}$, M atsumoto $\mathrm{T}$ et al.: Treatment of a full-thickness articular cartilage defect in the femoral condyle of an athlete with autologous bone-marrow stromal cells. 0 steoarthr. Cartil. 15, 226-231 (2007).

5. De U garte D A, M orizono K, Elbarbary A et al.: Comparison of multi-lineage cells from human adipose tissue and bone marrow. Cells Tissues O rgans 174, 101-109 (2003).

6. Georges $P, M$ uirhead RP, W illiams L et al.: Comparison of size, viability, and function of fetal pig islet-like cell clusters after digestion using collagenase or liberase. Cell Transplant. 11, 539-545 (2002).
7. Wang $\mathrm{H}$, Van Blitterswijk CA, Bertrand-D e H aas M, Schuurman AH , Lamme EN : Improved enzymatic isolation of fibroblasts for the creation of autologous skin substitutes. In Vitro Cell D ev. Biol. Anim. 40, 268-277 (2004).

- Comprehensively analyzes the effect of various Clostridium histolyticum collagenase preparations, the addition of neutral proteases and variable digestion periods in the isolation procedure for human fibroblasts. They demonstrate that the right balance in digestion parameters not necessarily lies where cell viability is highest or tissue digestion most efficient. The final and total cell outcome after cultivation is the main evaluation criteria not regarding the functionality of the cells.

8. Williams SK, M cKenney S, Jarrell BE: Collagenase lot selection and purification for adipose tissue digestion. Cell Transplant. 4, 281-289 (1995).

- The use of crude C lostridial collagenase in the digestion of human adipose tissue is evaluated with respect of cell yield and cell adherence. They convincingly point out lot-to-lot variation, test collagenase preparations subjected to different degrees of purification and prove that neutral proteases are essential for optimal digestion. They conclude that purified collagenase added to a specified protease will be optimal in applications where control and reproducibility are of the essence.

9. H ayman D M, Blumberg TJ, Scott CC, Athanasiou KA: The effects of isolation on chondrocyte gene expression. Tissue Eng. 12, 2573-2581 (2006).
10. Varma M J, Breuls RG, Schouten TE et al.: Phenotypical and functional characterization of freshly isolated adipose tissue-derived stem cells. Stem Cells D ev. 16, 91-104 (2007).

- Through triple-color fluorescence-activated cell sorting (FACS) analysis, this study demonstrates the applicability of the stromal vascular fraction (SVF) cells in engineering purposes involving bone repair. Freshly isolated SVF cells are compared with cultured adipose tissuederived stem cells (ASC s), and through critical examination it was shown that the loss of CD 34+ cells during culture was caused by a reduction in expression as opposed to an overgrowth of CD 34- cells.

11. M itchell JB, M clntosh K, Z vonic S et al.: I mmunophenotype of human adipose-derived cells: temporal changes in stromal-associated and stem cellassociated markers. Stem Cells 24, 376-385 (2006).

- $\quad$ Applying limited dilution assays and FACS analysis, this study elegantly demonstrates the selection of a homogeneous cell population through adherence of SVF cells and subsequent serial passaging. D uring expansion, ASC cultures are enhanced for stromal colony-forming cells with at least osteogenic and adipogenic capacity.

12. Sekiya I, Larson $B L$, Smith JR, Pochampally R, Cui JG, Prockop DJ: Expansion of human adult stem cells from bone marrow stroma: conditions that maximize the yields of early progenitors and evaluate their quality. Stem Cells 20, 530-541 (2002). 
13. Kin T, Johnson PR, Shapiro AM, Lakey JR: Factorsinfluencing the collagenase digestion phase of human islet isolation. Transplantation 83, 7-12 (2007).

14. Zuk PA, Zhu M, M izuno $H$ et al.: $M$ ultilineage cells from human adipose tissue: implications for cell-based therapies. Tissue Eng. 7, 211-228 (2001).

- $\quad$ Study forming the basic theory of presences and characteristics of ASC $s$ that states the basic definition of ASC s regarding differentiation potential and surface markers. The author healthily questions the existence of multipotential mesenchymal stem cells in adipose tissue as opposed to several different lineage precursor cells.

15. Guilak F, Lott KE, Awad H A et al.: Clonal analysis of the differentiation potential of human ASC s. J. Cell. Physiol. 206(1), 229-237 (2005).

- Investigation takes up the question raised by [14] and proves the presence of a real stem cell with multilineage potential in adipose tissue. Through ring-cloning expansion and differentiation assays, they show that ASC cultures contain cells of varying potential in adipogenic, osteogenic, chondrogenic and neurogenic differentiation.

16. M eyerrose TE, D e U garte $D A, H$ ofling AA et al.: In vivo distribution of human adipose-derived mesenchymal stem cells in novel xenotransplantation models. Stem Cells 25(1), 220-227 (2007).

17. Yoshimura K, Shigeura T, M atsumoto D et al.: Characterization of freshly isolated and cultured cells derived from the fatty and fluid portions of liposuction aspirates. J. Cell. Physiol. 208, 64-76 (2006).

18. Aust L, D evlin B, Foster SJ et al.: Yield of human adipose-derived adult stem cells from liposuction aspirates. Cytotherapy 6 , 7-14 (2004).

19. O edayrajsingh-Varma M J, van $\mathrm{H}$ am SM , Knippenberg $M$ et al.: Adipose tissuederived mesenchymal stem cell yield and growth characteristics are affected by the tissue-harvesting procedure. Cytotherapy 8 , 166-177 (2006).

20. Bellows CG, Aubin JE: D etermination of numbers of osteoprogenitors present in isolated fetal rat calvaria cells in vitro. Dev. Biol. 133, 8-13 (1989).
21. Wu X, Peters J M, G onzalez FJ, Prasad HS, Rohrer M D, Gimble JM : Frequency of stromal lineage colony forming units in bone marrow of peroxisome proliferatoractivated receptor- $\alpha$-null mice. Bone 26 , 21-26 (2000).

22. Castro-M alaspina $H, G$ ay $R E$, Resnick $G$ et al.: C haracterization of human bone marrow fibroblast colony-forming cells (CFU -F) and their progeny. Blood 56, 289-301 (1980).

23. Sen $A$, Lea-Currie YR, Sujkowska $D$ et al.: Adipogenic potential of human adipose derived stromal cells from multiple donors is heterogeneous. J. Cell. Biochem. 81, 312-319 (2001).

24. Gronthos S, Franklin D M, Leddy H A Robey PG, Storms RW, Gimble JM : Surface protein characterization of human adipose tissue-derived stromal cells. J. Cell. Physiol. 189, 54-63 (2001).

25. Katz AJ, Tholpady A, Tholpady SS, Shang $\mathrm{H}, \mathrm{O}$ gle RC: Cell surface and transcriptional characterization of human adipose-derived adherent stromal (hADAS) cells. Stem Cells 23, 412-423 (2005).

26. Daniels E: Cytori Therapeutics, Inc. Regen. M ed. 2(3), 317-320 (2007). 